Proc. Globecom Workshops, 6th IEEE International Workshop on Management of

Emerging Networks and Services, Austin, TX, 12-14 December 2014, pp. 207-212.

\title{
Opportunistic Wireless Charging for Mobile Social and Sensor Networks
}

\author{
Eyuphan Bulut \\ Cisco Systems \\ 2200 President George Bush Highway \\ Richardson, TX 75082 \\ ebulut@cisco.com
}

\author{
Mehmet Eren Ahsen \\ University of Texas, Dallas \\ 800 W. Campbell Road \\ Richardson, TX 75080 \\ ahsen@utdallas.edu
}

\author{
Boleslaw K. Szymanski \\ Rensselaer Polytechnic Institute \\ 110 8th St \\ Troy, NY 12180 \\ szymansk@cs.rpi.edu
}

\begin{abstract}
Even though sensor networks have been used in many applications, the limited capacity of the node batteries is still an obstacle in continuous usage of such networks. Recent advances in wireless energy transfer and rechargeable lithium batteries have opened new ways of solving this problem. In this paper, we study a charging mechanism for mobile social and sensor networks which consist of human-carried mobile devices. We investigate the problem of selecting a subset of nodes which will be responsible for charging the rest of the nodes in the network in such a way that all nodes will be able to continue to operate without any battery depletion. We provide a solution and evaluate its performance through simulations. The results show that the proposed approach can provide a fully operating network without any battery depletion problem if the node relations are similar in different cycles.
\end{abstract}

\section{INTRODUCTION}

Today, multiple devices (smartphones, GPS etc.) are equipped with sensors and there are many applications of wireless sensor networks (WSN) which require continuous functioning. However, these mostly battery powered devices can only function limited time. Even though their lifetime can be prolonged via various energy saving mechanisms or with the usage of more powerful batteries, this can only defer the end of their battery lifetime and cannot generate uninterrupted functioning networks.

With recent breakthroughs in the areas of wireless energy transfer [1], [2] and rechargeable lithium batteries [3], a new direction of research studies has been initiated for solving battery depletion problem in sensor networks. Wireless energy transfer is the transmission of electric energy from a power source to a receiver without a conductor. Even though it is a quite new technology and has still challenges like increasing charging efficiency to longer distances (i.e. the distance between the charging and charged device), it is a promising solution for continuously functioning sensor networks. Not only basic sensor nodes but also some mobile phones (e.g. Nokia's Lumia) and electric vehicles [4] have also been manufactured with built-in wireless charging capability. There are multiple devices offered by different companies and the charging time of such devices is comparable to wired connections. According to Business Week report [5], deployment of wirelessly charged devices is projected to increase to around 100 million by 2015 .
In this paper, we assume that there is a network of sensors located on mobile devices carried by humans. These devices can sense their environment via built-in sensors and communicate with other devices in their communication range. Military based sensor networks (with devices carried by soldiers in a field) or delay tolerant mobile phone networks (i.e., in which devices are connected via Bluetooth or $\mathrm{WiFi}$ ) can be considered as instances of such networks. We study the problem of wireless charging of the sensor nodes in such a network using the social relations of nodes (i.e., people carrying them). Assuming that there is no or minimal control over the mobility of nodes, we find out a subset of nodes in such networks that can be used to charge the rest of the devices such that all devices in the network can be kept functioning continuously without running out of battery. As an example scenario of this problem in practice, consider a military network, where several soldiers are in the field for an operation or monitoring purposes. They move around and encounter each other, some of them occasionally visit the army encamp for necessary supplies and go back to the field. In such a network, when the batteries of the sensor-equipped devices carried by soldiers deplete their energy, and substitute batteries are not available, one solution could be the replacement or recharging of such sensors by the other nodes who visit the army encamp regularly. Note that the encampment visiting schedule of the responsible nodes is important for system efficiency but so is the characteristics of the meeting history (i.e., social relations) of such nodes with non-visiting nodes. Thus, the analysis of the social relations between nodes is also significant. After the proposed algorithm analyzes the node relations, it identifies nodes which will be responsible for charging the rest of the devices in the network before they run out of battery. For evaluation of the proposed algorithm, we use two different real traces from different environments. Simulation results show that the selected set of charger nodes can charge the rest of nodes in the network in the subsequent cycles with high efficiency as the node relations in the network are regular and predictable.

The rest of the paper is organized as follows. We discuss the related work in Section II. In Section III, we define the system model and define the problem. We provide the details 
of proposed solution in Section IV. In Section V, we provide the simulation setting and evaluation of proposed system using real traces. Finally, we end up with conclusion and outline the future work in Section VI.

\section{RELATED WORK}

Wireless charging has been studied in sensor networks. It has been shown that energy problem in sensor networks can be solved by using mobile vehicles with high volume batteries acting as mobile chargers to deliver energy to sensors. In [6], authors design and implement a wireless charging system for a sensor network consisting of stable sensor nodes. [7] studies the optimization of path traveled by mobile charger nodes to provide enough power to sensor nodes to avoid battery depletion. In [8], authors study the impact of wireless charging on sensor network deployment and routing arrangement and formalize the deployment and routing problem. An interesting charging mechanism in which mobile chargers can even collaborate by transferring power between each other is presented in [9]. Then, a charging algorithm that can maximize the number of nodes (located in one-dimensional line) that can be charged together is presented. Different from previous work, this paper introduces an opportunistic charging mechanism in which all nodes are mobile and some subset of nodes can charge others. The selection of charger set is determined based on the node relations.

Mobile Social Networks (MSNs), which are a type of Delay Tolerant Networks (DTN), are of growing significance as a result of the rapid and wide spread use of various personal wireless devices (e.g., cell phones, GPS devices) among people and their surroundings. Since the intermittent connectivity between nodes causes lack of stable end-to-end path in these networks, recently, many studies focused on providing efficient solutions for the routing of messages in MSNs. Some studies used the social network metrics (similarity [10], ranking [11], friendship [12]) to offer better routing algorithms. Other problems like efficient content distribution [13] and security of the network [14] have been studied but wireless charging of nodes in such mobile networks has never been studied before.

\section{SySTEM MODEL}

We consider a network of sensor equipped mobile devices carried by people. These devices can communicate with each other when they are within the communication range of each other. We assume that the batteries of such mobile devices have two internal units which can be rechargeable separately. While the first unit of the battery is used for the operation (i.e., sensing) of the node, the second unit will be gathering power from the charger nodes (i.e., when there is a recharging opportunity). Once the first unit depletes all its energy, the second unit becomes active and the power for the operation of the node is supplied from the second unit. The first unit then switches the mode to gather power from the charger nodes. In such a system, we assume some of the nodes (we call them mobile charger set, $S_{m c}$ ) will be responsible for the charging of all nodes in the network. To achieve that, the nodes in $S_{m c}$ set will need to carry extra or more powerful batteries which will be loaded from a station (i.e., army encamp) they visit regularly. Assume that each unit of a regular node battery has $p$ power that lasts $L$ seconds on the average and will need $\beta$ seconds of charging to be fully recharged. Moreover, assume that each mobile charger will be able to charge $c$ regular nodes. Thus, the power of their extra battery will be $c p$ and they will need $c \beta$ seconds of contact with other nodes to fully transfer the power of this extra unit ${ }^{1}$.

Assume a system of $N$ nodes. The goal is to select the minimum number of nodes (as mobile chargers) out of $N$ nodes such that they will be responsible for recharging of others and no node will deplete its battery during a cycle. A cycle is assumed to be a day in our problem, however, it can be defined as a parameter. We assume that every node starts with a fully charged battery. Throughout the day, they receive power from charger nodes. Both types of nodes also consume energy from their batteries for their operations. At the end of the day (i.e., cycle), regular nodes need to have enough power in (the second unit of) their batteries to continue to the next cycle day without the necessity of charging their batteries by themselves. Thus, only the charger nodes will need to charge their batteries to be used (for operating and recharging others) in the next cycle. This system provides continuous functioning of sensor networks in which there is no opportunity for each node to reach power sources by itself before they run out of battery.

\section{Proposed ApproACH}

We define the network topology as a graph $G=(V, E) . V$ is the set of nodes in the network, and $E$ denotes the edges between these nodes. The weights of the edges are assigned as the total contact duration (which can be computed from collected encounter history and it represents the average value in some repeating cycle like a day) between the nodes it connects. If there is no contact between two nodes at all, no edge is assumed between their vertexes. Here, we consider two nodes to be in contact if they are close enough to each other such to achieve good charging efficiency.

We formulate the problem as follows. The goal is to find the minimum set of mobile chargers such that every other node in the network will have enough total contact time with them to be charged by them. The variables used are:

$$
\begin{aligned}
v_{i} & = \begin{cases}1 & \text { if node } i \text { is a mobile charger } \\
0 & \text { otherwise }\end{cases} \\
c_{i, j} & =\text { Total contact duration between nodes } i \text { and } j \\
u_{i, j} & =\text { Total contact duration that node } i \text { uses to charge node } j
\end{aligned}
$$

\footnotetext{
${ }^{1}$ We assume that the charging efficiency of mobile charger nodes is perfect, thus, the entire capacity of extra battery in a charger node can be transferred to regular nodes. We will look at the effect of charging efficiency on results in our future work.
} 


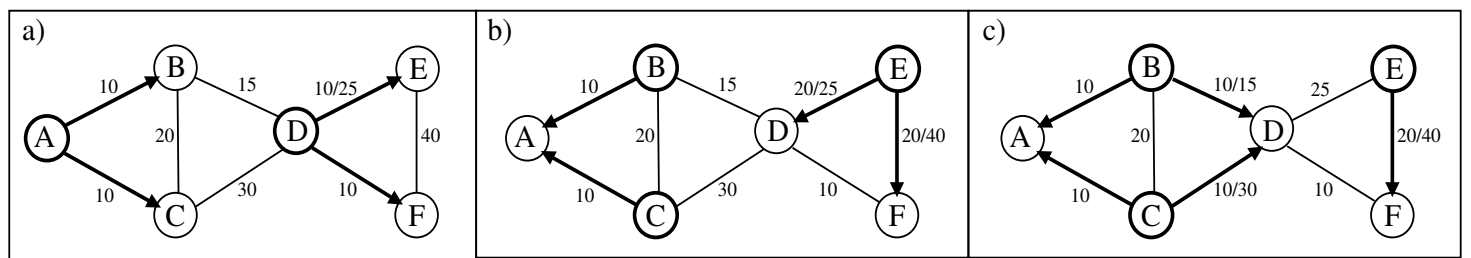

Fig. 1. Solutions on an example problem with a) $\left.\beta=10, c=2\left(S_{m c}=\{A, D\}\right), b\right) \beta=20, c=2\left(S_{m c}=\{B, C, E\}\right)$, and c) $\beta=20, c=1\left(S_{m c}=\{B, C, E\}\right)$.

Given the above variables ${ }^{2}$, the objective function is:

$$
\begin{aligned}
& \underset{\text { minimize }}{N} \sum_{i=1}^{N} v_{i} \\
& \text { subject to: } \\
& {[C 1] \sum_{i=1}^{N} u_{i j} \geq \beta\left(1-v_{j}\right), \forall 1 \leq j \leq N} \\
& {[C 2] \sum_{j=1}^{N} u_{i j} \leq c \beta \quad, \forall 1 \leq i \leq N} \\
& {[C 3] 0 \leq u_{i j} \leq c_{i j} v_{i} \quad, \forall 1 \leq i, j \leq N}
\end{aligned}
$$

The constraint [C1] ensures fulfillment of the total energy requirement of each node. If a node $j$ is selected to be a mobile charger, right hand side becomes zero, thus, that node does not require any power from other nodes. On the other hand, if it is not selected as a mobile charger, it requires at least $\beta$ seconds of contact duration (to be used in charging) with mobile charger nodes. The constraint [C2] ensures that for every node $j$ that is selected to be a mobile charger, the total amount of contact durations (used for charging) with the other nodes whom it is responsible to charge should be less than or equal to its maximum charging capacity. Finally, constraint [C3] forces each node $i$ whose non-zero contact duration with any other node $j$ is required to charge $j$ to be a mobile charger node with more contact time with $j$ than required to charge this node.

Assume we have $k$ mobile chargers. The total power that they can use to (re)charge other nodes in the network is $c p k$. The optimal number ${ }^{3}$ of mobile chargers then becomes:

$$
\begin{aligned}
c p k & \geq p(N-k) \\
k & \geq \frac{N}{c+1} \\
k & =\left\lceil\frac{N}{c+1}\right\rceil
\end{aligned}
$$

In the proposed system, some interesting situations may arise, like a mobile charger node with two different regular nodes in its range at the same time and it intends to charge both of them, or multiple mobile charger nodes getting in

\footnotetext{
${ }^{2}$ If the total contact duration between two nodes is longer than $\beta, c_{i, j}$ is set to $\beta$.

${ }^{3}$ This is given to show the optimal number that any solution including ILP can reach.
}

contact with the same node that they are assigned to charge. The former case is technically feasible as a single charger can charge multiple devices at the same time with similar performance. The latter might cause energy and efficiency loss. However, the probability that such cases will happen in such networks is very low since the connectivity between nodes is intermittent and having multiple connections is unlikely most of the time in practice.

In Figure 1, we show a sample problem and solutions with different $\beta$ and $c$ values. In the first case, $\beta=10$ and $c=2$, $A$ and $D$ are selected to be charger nodes. These nodes have at least 10 time units of contact duration with each of the other nodes they will charge and their maximum capacity of charging is not exceeded. Optimal number of mobile chargers $\left\lceil\frac{N}{c+1}\right\rceil=2$ is achieved with $\left|S_{m c}\right|=2$. In case b), the charger set consists of $B, C$ and $E$. This is because $\beta=20$ requires $A$ to be a charger or $B$ and $C$ to charge $A$. Either case requires a third charger node to be able to charge rest of the nodes. Thus, optimal number of mobile chargers is not feasible in this case and the feasible optimal number is 3 . In third case, setting $c=1$ does not change the mobile charger set, however, the list of nodes each charger node is responsible for charging (i.e., edges) changes. The solution in this case is also a good example where only some portion of total contact duration between two nodes is used for charging. Node $B$ charges node $D$ for only 10 time units out of its total of 15 time units of contact duration with $D$. This allows node $B$ to charge node $A$ too. Similarly, node $C$ can charge nodes $A$ and $D$.

\section{A. Greedy-Heuristic based Solution}

In this section we provide a greedy heuristic based solution to make it applicable in practice, regardless of complexity of exact solution. As illustrated in Algorithm 1, every node is assumed to be a charger at the beginning and a step by step removal procedure is applied. Until all nodes are processed, the minimum degree node among unprocessed charger nodes is found. Then, its link weight with other charger nodes is checked and tried to be fully charged by its neighbors. If it is possible, node is set as regular node. In the algorithm, the amount of maximum power (or the maximum contact duration needed) is determined according to the current status of charger and selected node. Among the three durations, namely, the maximum remaining power the node needs to be fully charged (maxNeeded), the maximum power that charger node can give to this node due to its total contact duration 
limitation (maxToThisNode) and the maximum remaining power that charger node can transfer to all nodes $(\operatorname{maxToAll})$, the minimum is found and the selected node is charged by the charger node during this duration. The complexity of the algorithm is $\mathrm{O}(\mathrm{V} \log \mathrm{V}+\mathrm{E})$, since each vertex and edge is processed once and minimum degree node can be found (line 12) in $\mathrm{O}(\log V)$. Note that, the order of processing the same degree nodes affects the performance of the greedy approach. For the example in Figure 1, greedy approach can give the illustrated solution if the algorithm starts processing from node $A$. Otherwise, it may yield different results.

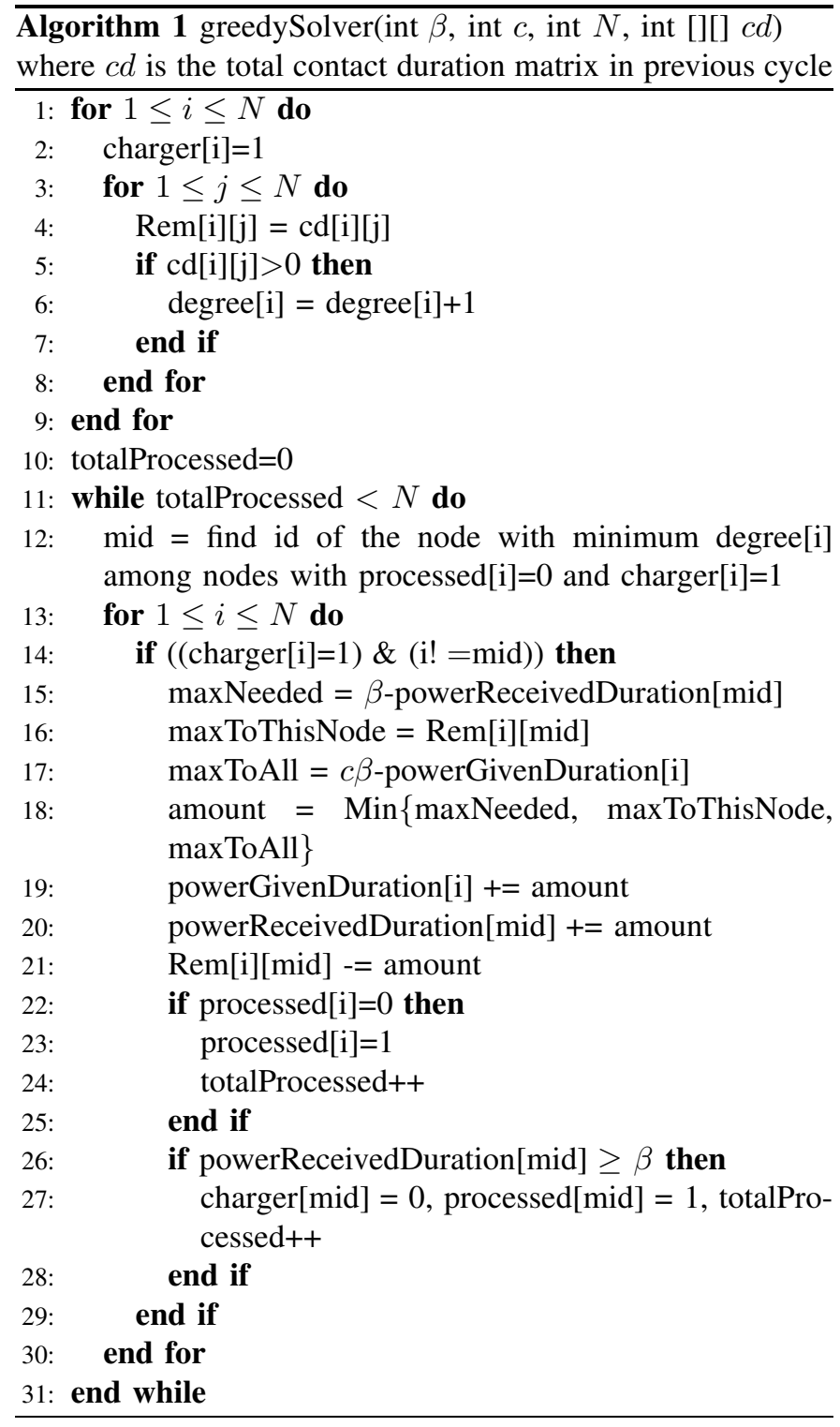

\section{B. Running of Opportunistic Wireless Charging $(O W C)$}

Once the set of charger nodes is determined, the charging process works as follows. To control the remaining contact time duration between each pair of nodes that will be used for charging, a variable, $R_{i j}$ is defined and initialized to $u_{i j}$, which is found by the algorithm. If the node $i$ is not charger node, it checks if the met node $j$ is responsible to charge it. If that is the case and if there is still some charging required by node $j$, node $j$ starts to charge the second battery unit of node $i$ until it is full or until it gets out of the range of node $i$. If node $i$ is the charger node and still needs to transfer more power to met node $j$, node $i$ starts to charge second battery of node $j$ until required power is transferred or nodes go out of their ranges. When a node leaves from the meeting point, it first updates the total contact duration information in current cycle (to be used for $S_{m c}$ decision in the next cycle) then stops charging the left node if it was charging.

\section{Simulations}

We evaluated the performance of proposed solutions using simulations. As a case study, we used social network traces of real objects carried by people and assume that once the set of mobile charger nodes are determined, they can be directed to a center to be loaded with extra power units and use them for recharging of other nodes. The simulator uses the traces of real objects from datasets collected from real MSN environments. The network parameters (number of nodes, etc.) are defined by the traces used.

\section{A. DataSets and Simulation Setting}

We used the following two real traces from the crawdad archive:

- Cambridge Dataset [15] includes Bluetooth sightings between iMotes carried by Cambridge University students.

- MIT reality data set consists of the traces of Nokia smart phones that were carried by MIT students and staff over nine months. In our simulations, we used the contacts logged during a three month period from the beginning of February to the end of April. The time frame covers the second academic semester where human relations are relatively stable and participants are mostly active on campus.

Before using the above datasets, we performed some preprocessing. Some nodes had very few contacts logged with other nodes and some nodes had very short contact durations (some logs were even showing the same start and end time for a meeting). To increase the number of contacts between nodes, we merged several days of data to one day and removed some overlapping contact durations between the same nodes. After these changes, we selected 45 nodes from MIT traces and 26 nodes from Cambridge traces to work with more reasonable set of contacts than original one.

\section{B. Simulation Results}

Given the set of contacts from a dataset, each node computes its total contact duration with other nodes in a day and the corresponding graph topology is formed. The mobile charger node set is then determined using the proposed solutions.

We define two performance metrics; i) number of mobile charger nodes selected (solved by CPLEX [16] for ILP solution with constraints defined in the beginning of Section IV) 


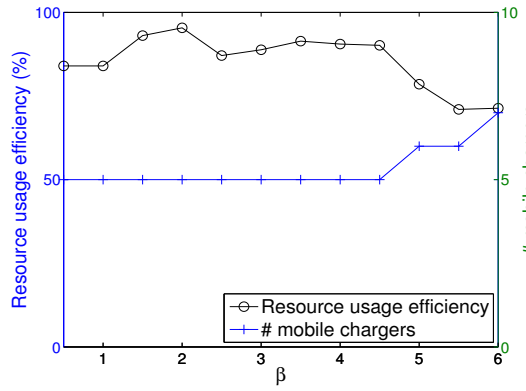

(a) Cambridge Dataset

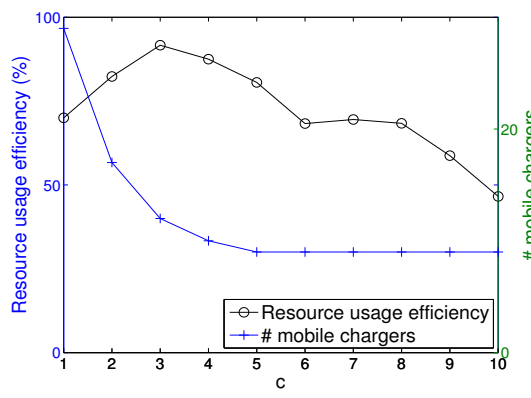

(d) MIT Dataset

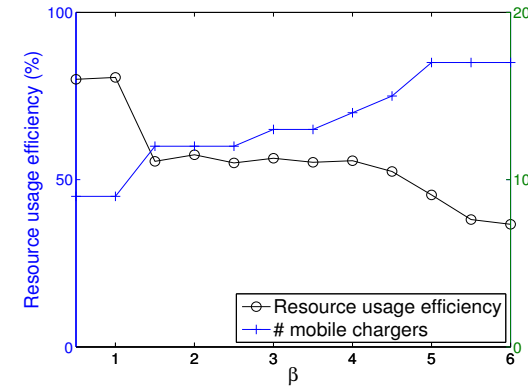

(b) MIT Dataset

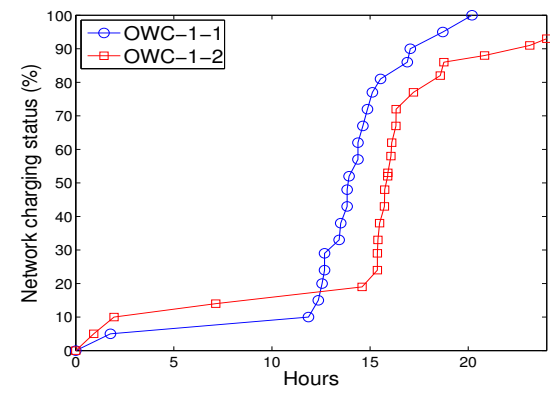

(e) Cambridge Dataset

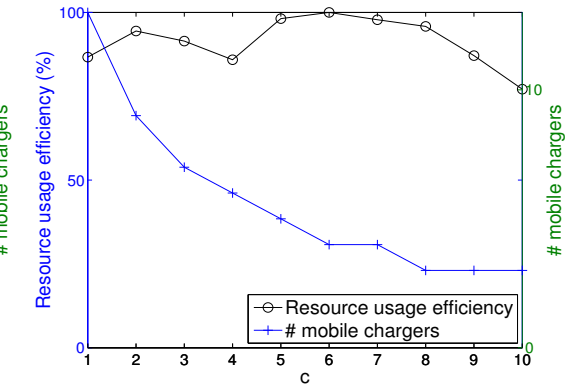

(c) Cambridge Dataset

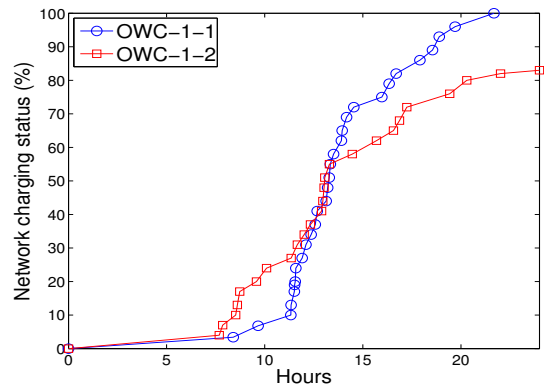

(f) MIT Dataset

Fig. 2. (a-b) Impact of $\beta$ (hours required for full charge) (c-d) Impact of $c$. (e-f) Opportunistic Wireless Charging (OWC) results with OWC- $x-y$ indicates that set of mobile chargers $\left(S_{m c}\right)$ is obtained from $x^{t h}$ part of the data and the OWC algorithm run on the $y^{t h}$ part of the data.

and ii) charger node set average resource usage efficiency, which is defined as the fraction of the total capacity of the (second) batteries of assigned charger nodes used for charging.

We first look at the impact of $\beta$ (when $c=5$ ) on these two metrics. Figure 2-a-b shows the results for $\beta$ ranging from 30 minutes to 6 hours in both datasets. In general, in both datasets, as the $\beta$ increases, the minimum number of mobile charger nodes required increases and the resource usage efficiency decreases since most of the mobile chargers' power becomes underutilized ${ }^{4}$.

Figure $2 \mathrm{c}$-d shows the impact of $c$ (when $\beta=1$ hour) which defines the maximum charging capacity of each mobile charger node on the two performance metrics. As expected, as $c$ increases, the number of mobile charger nodes required in the system decreases. However, the resource usage efficiency stays around the same value until some $c$ in both datasets. This is because not every node is a neighbor of all other nodes and therefore may not have a contact opportunity to charge them. Thus, excessive capacity of mobile charger nodes is underutilized and the resource usage efficiency starts to decrease after these boundary $c$ values. For Cambridge dataset, average resource usage efficiency (before boundary $c=8$ ) is $92 \%$ and for MIT dataset it is $80 \%$ (before boundary $c=5$ ).

Next, we look at how the greedy algorithm performs with respect to ILP solution. As Table I shows, greedy algorithm requires $34 \%$ more mobile chargers (average of the results

\footnotetext{
${ }^{4}$ In future work, we will investigate if the collaboratively working charger nodes, which can transfer power to each other, can increase the resource usage efficiency.
}

with $1 \leq c \leq 10$ and $\beta=1$ hour) yielding $21 \%$ decrease in $S_{m c}$ 's resource usage efficiency in Cambridge dataset. In MIT dataset, these numbers are higher compared to Cambridge because the node relations in Cambridge are more regular and the nodes have more neighbors in Cambridge dataset. In our future work, we will try to improve the performance of greedy algorithm.

\begin{tabular}{|c|l|l|}
\hline & $\begin{array}{l}\text { Increase in \# mobile } \\
\text { chargers }\end{array}$ & $\begin{array}{l}\text { Decrease in resource } \\
\text { usage efficiency }\end{array}$ \\
\hline \hline Cambridge & $34 \%$ & $21 \%$ \\
\hline MIT & $56 \%$ & $29 \%$ \\
\hline
\end{tabular}

TABLE I

PERFORMANCE OF GREEDY ALGORITHM

To see how the status of batteries change, we also run the opportunistic wireless charging (OWC) process on the network. To this extend, we first run OWC on the same dataset from which the set of mobile chargers, $S_{m c}$, is determined. Then, we determine $S_{m c}$ from the encounter history between nodes only in the first half of dataset and run OWC using the traces in the second half of the dataset. As the plots in Figure 2e-f shows, in OWC-1-1 case, by the end of the day (i.e. charging cycle), as expected, second batteries of all regular nodes can collect enough power to cover their needs during the next cycle. In OWC-1-2 case, $S_{m c}$ is computed using the traces in first part but the OWC is run using the traces in second part. In Cambridge traces, $94 \%$ of total capacity in second batteries of all regular nodes is charged while in MIT 


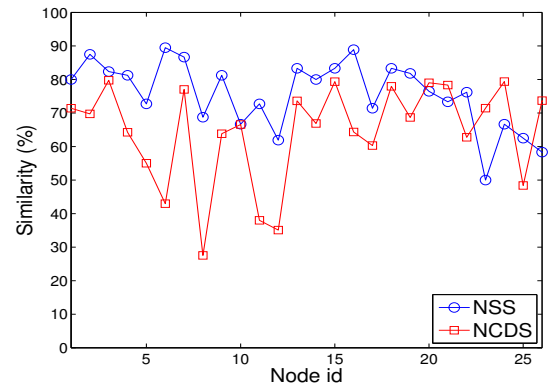

(a) Cambridge Dataset

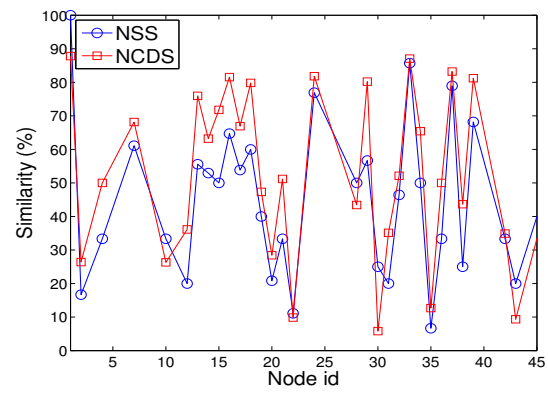

(b) MIT Dataset

Fig. 3. Similarity of first and second half of two data.

traces, only $82 \%$ of total capacity is charged.

To analyze the OWC-1-2 performance difference in both datasets, we also did a comparison between the first and second half of them. We define two different similarity metrics:

Neighbor Set Similarity (NSS) shows the similarity of neighbor set of nodes. Let $N_{1}(i)$ and $N_{2}(i)$ denote the neighbor set of node $i$ in first and second half of the data, respectively. Then, we formulate the neighbor set similarity as:

$$
N S S=1-\sum_{i=1}^{N} \frac{\left|N_{1}(i)-N_{2}(i)\right|}{N}
$$

Neighbor Contact Duration Similarity (NCDS) shows the similarity of the contact duration between all pairs in two different parts of the data. Let $c_{y}(i, j)$ denote the total contact duration between node $i$ and $j$ in part $y$. Then, (weighted) NCDS is formulated as:

$$
N C D S=1-\frac{\sum_{i=1, j \neq i}^{N}\left|c_{1}(i, j)-c_{2}(i, j)\right|}{\sum_{i=1, j \neq i}^{N}\left|c_{1}(i, j)+c_{2}(i, j)\right|}
$$

Figure 3 shows the introduced similarity values of nodes in both datasets. In Cambridge dataset, the average NSS value for all nodes is $77 \%$, while it is $46 \%$ in MIT traces. Similarly, the average NCDS is $64 \%$ in Cambridge traces, while it is $54 \%$ in MIT traces. These results clearly explain the results in Figure 2e-f. For networks with higher regularity (yielding higher NSS and NCDS similarity in different parts of the data) in node relations, $S_{m c}$ computed from a training data (i.e., first half) can be used to charge nodes in next cycle (i.e., second half).

\section{CONCLUSION}

In this paper we studied opportunistic wireless charging (OWC) problem in mobile social and sensor networks and specifically investigated the selection of some nodes as mobile charger nodes such that they will be responsible for the charging of other nodes. Our goal was to create a network in which the burden of charging stays only in limited number of nodes. Simulation results on real MSN traces demonstrate that if the node relations show similar pattern through different cycles, OWC can be used to charge other nodes in the network.

In our future work, we will extend our algorithm to include the regularity of node relations. Even though the ILP solution can find $S_{m c}$ with optimum number of mobile chargers, it is also possible that multiple $S_{m c}$ sets with the same $k=$ $\left\|S_{m c}\right\|$ may exist. If the selection of nodes in $S_{m c}$ can be made considering the regularity of relations of nodes in $S_{m c}$ with others, the system can achieve better efficiency in the future cycles. We will also update the $S_{m c}$ selection algorithm to take into account predictability and similarity of node relations.

\section{REFERENCES}

[1] A. Kurs, A. Karalis, R. Moffatt, J. D. Joannopoulos, P. Fisher, and M. Soljacic, Wireless power transfer via strongly coupled magnetic resonances, Science, vol. 317, no. 5834, pp. 8386, 2007.

[2] B. Cannon, J. Hoburg, D. Stancil, and S. Goldstein, Magnetic resonant coupling as a potential means for wireless power transfer to multiple small receivers, IEEE Trans. on Power Electronics, vol. 24, no. 7, pp. 1819 1825, July 2009.

[3] K. Kang, Y. S. Meng, J. Breger, C. P. Grey, and G. Ceder, Electrodes with high power and high capacity for rechargeable lithium batteries, Science, vol. 311, no. 5763, pp. 977980, 2006.

[4] Wireless charging for electric vehicles, http://www.hevopower.com, accessed on 4/1/2013.

[5] O. Kharif, The Coming War Over Wireless Charging, url: http://www.businessweek.com/articles/2012-11-08/the-coming-warover-wireless-charging, accessed on 01/28/2013.

[6] Y. Peng, Z. Li, W. Zhang, and D. Qiao, Prolonging sensor network lifetime through wireless charging, in IEEE RTSS 2010.

[7] Y. Shi, L. Xie, Y. Hou, and H. Sherali, On renewable sensor networks with wireless energy transfer, in IEEE INFOCOM 2011.

[8] B. Tong, Z. Li, G. Wang, and W. Zhang, How wireless power charging technology affects sensor network deployment and routing, in IEEE ICDCS 2010.

[9] S. Zhang, J. Wu, S. Lu, Collaborative Mobile Charging for Sensor Networks, In Mobile Adhoc and Sensor Systems (MASS), 2012 IEEE 9th International Conference on (2012), IEEE, pp. 8492.

[10] E. Daly and M. Haahr, Social Network Analysis for Information Flow in Disconnected Delay-Tolerant MANETs, in IEEE Transactions on Mobile Computing, vol. 8(5), May, 2009.

[11] P. Hui, J. Crowcroft, and E. Yoneki, BUBBLE Rap: Social Based Forwarding in Delay Tolerant Networks, In Proc. of ACM MobiHoc, 2008.

[12] E. Bulut, and B. Szymanski, Exploiting Friendship Relations for Efficient Routing in Mobile Social Networks, in IEEE Transactions on Parallel and Distributed Systems (TDPS), 2012.

[13] X. Zhuo, Q. Li, G. Cao, Y. Dai, B. Szymanski, and T. L. Porta, Social-Based Cooperative Caching in DTNs: A Contact Duration Aware Approach, in Proc. of IEEE MASS, 2011.

[14] E. Bulut, and B. Szymanski, Secure Multi-copy Routing in Compromised Delay Tolerant Networks, Wireless Personal Communications (2012): 120 , December 15, 2012.

[15] J. Leguay, A. Lindgren, J. Scott, T. Friedman, J. Crowcroft and P. Hui, CRAWDAD data set upmc/content (v. 2006-11-17), downloaded from http://crawdad.cs.dartmouth.edu, 2006.

[16] IBM ILOG CPLEX Optimizer, http://www-01.ibm.com/software /integration/optimization/cplex-optimizer/, accessed on 07/04/2012. 